\title{
Certain representations of the Cuntz relations, and a question on wavelets decompositions
}

\author{
Palle E.T. Jorgensen
}

\begin{abstract}
We compute the Coifman-Meyer-Wickerhauser measure $\mu$ for certain families of quadrature mirror filters (QMFs), and we establish that for a subclass of QMFs, $\mu$ contains a fractal scale.
\end{abstract}

\section{Introduction}

It is known that certain representations of the Cuntz relations (or equivalently the Cuntz algebras $\mathcal{O}_{N}[\underline{6}$ serve as a computational tool for wavelet analysis. The reason for this is that a representation of the Cuntz relations on a Hilbert space $\mathcal{H}$ induces a successive subdivision of $\mathcal{H}$ into orthogonal subspaces. To get the scaling feature of wavelets enter into our representations, build a family of operators from the endomorphism $z \longrightarrow z^{N}$ on the circle $\mathbb{T}$, and $N$ carefully chosen multiplication operators on $L^{2}(\mathbb{T})$ with respect to Haar measure on $\mathbb{T}$. In the dyadic case, we work with $z \longrightarrow z^{2}$ and with two multiplication operators, i.e., two functions on $\mathbb{T}$. In the language of signal processing, these functions $m_{0}$, and $m_{1}$ are called low-pass and high-pass filters, respectively; or the pair is called a quadrature-mirror system of filters. By a standard procedure [7], we construct the scaling function (or father function) $\varphi$ on $\mathbb{R}$ from $m_{0}$, and the wavelet function (or mother function) $\psi$ from $m_{1}$. We get two corresponding isometries which yield a representation of the Cuntz algebra $\mathcal{O}_{2}$. These representations have been studied in earlier papers, see e.g., 1, 2, 3, 4, 8, 12. The problem in wavelet theory is to build orthonormal bases in $L^{2}(\mathbb{R})$ from these data. This can be done [7], and the wavelet bases have advantages over the earlier known basis constructions, one of the main advantages is efficiency of computation.

2000 Mathematics Subject Classification. 42C40; 42A16; 43A65; 42A65.

Key words and phrases. Hilbert space, Cuntz algebra, map, wavelet packets, pyramid algorithm, product measures, orthogonality relations, equivalence of measures, iterated function systems (IFS), scaling function, multiresolution, subdivision scheme, singular measures, absolutely continuous measures.

This material is based upon work supported by the U.S. National Science Foundation under Grant No. DMS-0139473 (FRG). 
While choice pyramids (see e.g., 15 and 16 ) have been much used in computations already, the presence of representations of $\mathcal{O}_{N}$ yields a Hilbert space geometric way of realize such combinatorial pyramids. Representing the integers $\mathbb{N}_{0}=\{0,1,2, \ldots\}$ using the Euclidean algorithm, and using a corresponding pyramid, we get a orthogonal basis for $L^{2}(\mathbb{R}), \varphi_{0}, \varphi_{1}, \varphi_{2}, \ldots$ called a wavelet packet. A wavelet packet decomposition arises by a combinatorial selection of a subset of the index set $\mathbb{N}_{0}$ for these basis function, and a corresponding subset of the scaling operations $x \longrightarrow N^{j} x$ (i.e., a selection of a subset of the integers $\mathbb{Z}$ ) such that this selection of subsets yields an orthonormal basis for $L^{2}(\mathbb{R})$. As pointed out in [15] and [16, the selection of such feasible pairs of subsets involves a combinatorial tiling question, and a problem in measure theory. In this paper we show how these questions may conveniently be addressed with the use of the non-commutative harmonic analysis coming from the theory of representations of the $C^{*}$-algebra $\mathcal{O}_{N}$.

And in the course of the paper, we show that the scope of our method is wider than the original context of standard wavelets: It applies equally well to the class of fractals that arise from affine iterated function systems (IFS); see e.g., [8], 10, and [1].

Wavelets and wavelet packets are orthonormal bases, or Parseval frames, for the Hilbert space $L^{2}(\mathbb{R})$ which are built from a small set of generating functions by applying only two operations to them, translation by the integers $\mathbb{Z} \subset \mathbb{R}$, and by dyadic scaling $t \longrightarrow 2^{j} t, j \in \mathbb{Z}$. The best known case is that of singly generated wavelets. Then there is a function $\psi \in L^{2}(\mathbb{R})$ such that the family

$$
\left\{2^{j / 2} \psi\left(2^{j} t-k\right)\right\}_{j, k \in \mathbb{Z}}
$$

is an orthonormal basis for $L^{2}(\mathbb{R})$. Wavelet packets (in the orthonormal case) are sequences $\left(\varphi_{n}\right)_{n \in \mathbb{N}_{0}}$ and subsets $J \subset \mathbb{N}_{0} \times \mathbb{Z}$ such that the two families

$$
\left\{2^{j / 2} \varphi_{n}\left(2^{j} t-k\right) \mid(n, j) \in J, k \in \mathbb{Z}\right\}
$$

and

$$
\left\{\varphi_{n}(t-k) \mid n \in \mathbb{N}_{0}, k \in \mathbb{Z}\right\}
$$

are both both orthonormal bases.

In the seminal paper [5], the authors Coifman, Meyer, and Wickerhauser proposed a selection of systems (1.2) from (1.3); the idea being that (1.3) may be constructed by an effective and relatively simple algorithm. The construction starts with a quadrature-mirror wavelet filter, i.e., a Fourier series

$$
m_{0}(x)=\sum_{k \in \mathbb{Z}} a_{k} e^{-i 2 \pi k x}
$$

such that

$$
\sum_{k \in \mathbb{Z}} \overline{a_{k}} a_{k+2 \ell}=\delta_{0, \ell}
$$


and

$$
\sum_{k \in \mathbb{Z}} a_{k}=\sqrt{2}
$$

If the expansion in (1.4) is a finite sum, then the functions $\varphi_{0}, \varphi_{1}, \varphi_{2}, \cdots$ will be of compact support. The advantage with using (1.2) over (1.1) or (1.3) is that the basis functions in (1.2) are better localized in time-frequency, as is spelled out in [5] and $\mathbf{1 5}$.

The authors of [5] propose a certain integral decomposition of $L^{2}(\mathbb{R})$ which is based on a certain measure $\mu_{0}$ on the unit-interval $[0,1]$. The idea is the following: The quadrature rules (1.5) lead to a dyadic decomposition of the Hilbert space $\ell^{2}(\mathbb{Z})$, repository for the wavelet coefficients: For each $k \in \mathbb{N}$, and each dyadic interval

$$
\left[\xi, \xi+2^{-k}\right), \xi=\frac{i_{1}}{2}+\cdots+\frac{i_{k}}{2^{k}}
$$

we assign a closed subspace $\mathcal{H}(\xi) \subset \ell^{2}$, such that the $2^{k}$ distinct subspaces $\mathcal{H}(\xi)$ are mutually orthogonal, and

$$
\sum_{\xi}^{\oplus} \mathcal{H}(\xi)=\ell^{2}
$$

If $e_{0}$ denotes the vector $e_{0}(j):=\delta_{0, j}$ in $\ell^{2}$, and $P(\xi)$ the orthogonal projection onto $\mathcal{H}(\xi)$, then the measure $\mu_{0}$ is determined by

$$
\mu_{0}\left(\left[\xi, \xi+2^{-k}\right)\right)=\left\|P(\xi) e_{0}\right\|^{2}
$$

where $\xi$ is the dyadic rational (1.7).

More generally, if $f \in \mathcal{H},\|f\|^{2}=1$, we set $\mu_{f}(\cdot)=\|P(\cdot) f\|^{2}$, and note that each $\mu_{f}$ is a probability measure. These measures are shown in section 3 to dictate the wavepacket analysis in the Hilbert space $L^{2}(\mathbb{R})$.

The expectation is that $\mu_{0}$ is absolutely continuous with respect to the Lebesgue measure on $[0,1]$. We give a formula for $\mu_{0}$ directly in terms of the coefficients $\left(a_{j}\right)_{j \in \mathbb{Z}}$ in (1.4), and we give a one-parameter family of coefficients $a_{j}(\beta)$ where $\beta$ parameterizes the circle, and where for $|\beta|<\pi / 4$, the measure $\mu_{0}$ on "small" dyadic intervals $J$ is governed by the formula

$$
\mu_{0}(\text { dyadic interval } J) \approx|J|^{s},
$$

where $|J|$ denotes the length of $J$, and where

$$
s=\frac{\ln \left(a_{0}(\beta)^{-2}\right)}{\ln 2} .
$$

In the interval for $\beta$, the coefficients $a_{j}(\beta)$ are real-valued, and when $|\beta|<\pi / 4$, $a_{0}(\beta)>1 / \sqrt{2}$. 


\section{Fourier Polynomials}

Let $\mathbb{T}=\{z \in \mathbb{C}|| z \mid=1\}$, and let $D \in \mathbb{N}$. Consider the following two functions

$$
m_{0}(z)=\sum_{k=0}^{2 D-1} a_{k} z^{k}
$$

and

$$
\begin{aligned}
m_{1}(z) & =z^{2 D-1} \overline{m_{0}(-z)} \\
& =\sum_{k=0}^{2 D-1}(-1)^{k} \bar{a}_{2 D-1-k} z^{k}
\end{aligned}
$$

We shall need several lemmas.

LEMma 2.1. The finite sequence $a_{0}, a_{1}, \cdots, a_{2 D-1}$ in (2.1) satisfies

$$
\sum_{j \in \mathbb{Z}} \bar{a}_{k} a_{k+2 \ell}=\delta_{0, \ell}
$$

if and only if anyone of the following equivalent conditions holds:

(a) The matrix function

$$
U(z)=\sum_{k=0}^{D-1} A_{k} z^{k}, z \in \mathbb{T}
$$

is unitary, where the coefficient matrices are

$$
A_{k}=\left(\begin{array}{cc}
a_{2 k} & a_{2 k+1} \\
a_{2(D-k)-1} & -a_{2(D-k-1)}
\end{array}\right) \text { for } k=0,1, \cdots, D-1 .
$$

(b) The operators

$$
\left(S_{i} f\right)(z)=m_{i}(z) f\left(z^{2}\right), f \in L^{2}(\mathbb{T}), i=0,1,
$$

and their adjoints $S_{i}^{*}$ satisfy

$$
\left\{\begin{array}{c}
S_{i}^{*} S_{j}=\delta_{i, j} I, i, j=0,1 \\
S_{0} S_{0}^{*}+S_{1} S_{1}^{*}=I
\end{array} .\right.
$$

(c) The following matrix function

$$
M(z):=\frac{1}{\sqrt{2}}\left(\begin{array}{cc}
m_{0}(z) & m_{0}(-z) \\
m_{1}(z) & m_{1}(-z)
\end{array}\right), z \in \mathbb{T},
$$

is unitary, i.e., $M(z)^{*} M(z)=I$, for all $z \in \mathbb{T}$.

REMARK 2.2. In the summation (2.3), it is understood that the terms are zero if the summation index is not in the range which is specified for the sequence: If for example $D=2$, then the conditions (2.3) spell out as follows:

$$
\left\{\begin{array}{c}
\left|a_{0}\right|^{2}+\left|a_{1}\right|^{2}+\left|a_{2}\right|^{2}+\left|a_{3}\right|^{2}=1 \\
\bar{a}_{0} a_{2}+\bar{a}_{1} a_{3}=0
\end{array} .\right.
$$


The interpretation of (2.4) is that the matrices $A_{0}, A_{1}, \cdots A_{D-1}$ are Fourier coefficients for a matrix function $z \longrightarrow U(z)$ defined on $\mathbb{T}$. The requirements is that for each $z \in \mathbb{T}$, the 2 by 2 matrix $U(z)$ is unitary, i.e., that

$$
U(z)^{*} U(z)=I, z \in \mathbb{T} \text {. }
$$

In general, for a function of the form (2.4), the unitarity condition (2.10) is equivalent to the following conditions on the constant matrices $A_{0}, A_{1}, \cdots$ :

$$
\sum_{k} A_{k+n} A_{k}^{*}=\delta_{0, n}
$$

or equivalently

$$
\sum_{k} A_{k}^{*} A_{k+n}=\delta_{0, n}
$$

Again if $D=2$, these conditions flesh out as follows: The two matrices

$$
A_{0}=\left(\begin{array}{cc}
a_{0} & a_{1} \\
a_{3} & -a_{2}
\end{array}\right) \text { and } A_{1}=\left(\begin{array}{cc}
a_{2} & a_{3} \\
a_{1} & -a_{0}
\end{array}\right)
$$

satisfy

$$
\left\{\begin{array}{c}
A_{0} A_{0}^{*}+A_{1} A_{1}^{*}=I \\
A_{1} A_{0}^{*}=0
\end{array} .\right.
$$

Proof. of Lemma 2.1. The coefficients $a_{0}, a_{1}, \cdots, a_{2 D-1}$ are given and the two functions $m_{0}(z)$ and $m_{1}(z)$ are defined as in (2.1) and (2.2). Even though they are defined for all $z \in \mathbb{C}$, we shall use only the restrictions to $\mathbb{T}$. It is convenient in some later calculations to introduce the substitution $z:=$ $e^{-i 2 \pi x}$; and with this substitution we shall also view $m_{0}$ and $m_{1}$ as one-periodic functions on the real line $\mathbb{R}$, and make the identification $\mathbb{T} \cong \mathbb{R} / \mathbb{Z}$. We now turn to the implications: 2.3$) \Longrightarrow($ a). We already noted that the unitarity of the function $U(z)$ in (2.4) may be expressed by the conditions (2.11). When (2.5) is substituted in 2.11), it is immediate that the two identities (2.3) and (2.11) are equivalent. (a) $\Longrightarrow$ (b). Using the normalized Haar measure $\mu$ on $\mathbb{T}$ and the corresponding inner product

$$
\langle f \mid g\rangle:=\int_{\mathbb{T}} \overline{f(z)} g(z) d \mu(z)
$$

of the Hilbert space $L^{2}(\mathbb{T})$, we get the formula

$\left(S_{i}^{*} f\right)(z)=\frac{1}{2} \sum_{w \in \mathbb{T}, w^{2}=z} \overline{m_{i}(w)} f(w)$, defined for $f \in L^{2}(\mathbb{T}), z \in \mathbb{T}$, and $i=0,1$.

As a result, we get the formula

$$
\left(S_{i}^{*} S_{j} f\right)(z)=\frac{1}{2} \sum_{w \in \mathbb{T}, w^{2}=z} \overline{m_{i}(w)} m_{j}(w) f(z) ;
$$


which states that the four operators $S_{i}^{*} S_{j}$ are multiplication operators. Hence the first part of (2.7) reads:

$$
\frac{1}{2} \sum_{w \in \mathbb{T}, w^{2}=z} \overline{m_{i}(w)} m_{j}(w)=\delta_{i, j}, z \in \mathbb{T} .
$$

Substitution of (2.1) into (2.18) shows that these conditions amount to the same sum rules (2.3), or equivalently (2.11). The second equation from (2.7) may be restated as

$$
\left\|S_{0}^{*} f\right\|^{2}+\left\|S_{1}^{*} f\right\|^{2}=\|f\|^{2}, \text { for all } f \in L^{2}(\mathbb{T}) ;
$$

or equivalently

$$
\sum_{i=0}^{1}\left|\frac{1}{2} \sum_{w^{2}=z} \overline{m_{i}(w)} f(w)\right|^{2} d \mu(z)=\int_{\mathbb{T}}|f(z)|^{2} d \mu(z) .
$$

But the summation on the left-hand sides fleshes out as follows:

$$
\begin{aligned}
& \frac{1}{4} \sum_{i=0}^{1} \sum_{w^{2}=\zeta^{2}=z} \overline{m_{i}(w)} m_{i}(\zeta) f(w) \overline{f \overline{(\zeta)}} \\
& =\frac{1}{2} \sum_{w^{2}=\zeta^{2}=z}\left(\frac{1}{2} \sum_{i=0}^{1} \overline{m_{i}(w)} m_{i}(\zeta)\right) f(w) f \overline{(\zeta)} \\
& =\frac{1}{2} \sum_{w^{2}=\zeta^{2}=z} \delta_{w, \zeta} f(w) f \overline{(\zeta)} \\
& =\frac{1}{2} \sum_{w^{2}=z}|f(w)|^{2}
\end{aligned}
$$

and

$$
\int_{\mathbb{T}} \frac{1}{2} \sum_{w^{2}=z}|f(w)|^{2} d \mu(z)=\int_{\mathbb{T}}|f(z)|^{2} d \mu(z)
$$

by an elementary property of the Haar measure. This proves $(a) \Longrightarrow(b)$; and in fact equivalence. (b) $\Longrightarrow(\mathrm{c})$. This implication also goes in both directions, and it is implicit in the previous step where we showed that the identities (2.18) are equivalent to (2.3). But an inspection of matrix entries shows that (2.18) is a restatement of the unitary property for the matrix $M(z)$ in (c).

Corollary 2.3. Consider a Fourier polynomial

$$
m(z)=\sum_{k=0}^{2 D-1} a_{k} z^{k}
$$

as in Lemma 2.1 and let $S=S_{m}$ be the operator on $L^{2}(\mathbb{T})$ defined by

$$
(S f)(z)=m(z) f\left(z^{2}\right), z \in \mathbb{T} .
$$

Then the following conditions are equivalent:

(i) $S$ is an isometry; 
(ii) $|m(z)|^{2}+|m(-z)|^{2}=2$, for $z \in \mathbb{T}$; and

(iii) $\sum_{k} \bar{a}_{k} a_{k+2 \ell}=\delta_{0, \ell}$.

Proof. The equivalence (i) $\Longleftrightarrow$ (ii) is immediate from an inspection of (2.18) in the special case $i=j=0$. To see (ii) $\Longleftrightarrow($ iii), it is simplest to write out the Fourier coefficients of the function

$$
z \longrightarrow \frac{1}{2} \sum_{w \in \mathbb{T}, w^{2}=z}|m(w)|^{2}
$$

A substitution of (2.22) into (2.24) yields

$$
\begin{aligned}
& \frac{1}{2} \sum_{j} \sum_{k} \sum_{w^{2}=z} \bar{a}_{j} a_{k} w^{k-j} \\
& =\sum_{n} \sum_{j, k \text { s.t. } k-j=2 n} \bar{a}_{j} a_{k}\left(\frac{1}{2} \sum_{w^{2}=z} w^{2 n}\right) \\
& =\sum_{n}\left(\sum_{j} \bar{a}_{j} a_{j+2 n}\right) z^{n},
\end{aligned}
$$

and it follows that the sum in (2.24) is the constant function 1 if and only if (iii) is satisfied. Note that (iii) is a restatement of condition (2.3) in Lemma 2.1 In the following we consider operators $S$ on the form $(S f)(z)=m(z) f\left(z^{2}\right)$, $z \in \mathbb{T}, f \in L^{2}(\mathbb{T})$, and their adjoints. But we will need these operators realized explicitly in the sequence space $\ell^{2} \cong L^{2}(\mathbb{T})$.

LEMMA 2.4. .

(a) The matrix representation for the operator $S$ in Corollary 2.3 relative to the standard basis $e_{n}(z):=z^{n}, n \in \mathbb{Z}$, for $L^{2}(\mathbb{T})$, is

$$
(S \xi)_{n}=\sum_{k} a_{n-2 k} \xi_{k}
$$

and the adjoint $S^{*}$ is

$$
\left(S^{*} \xi\right)_{n}=\sum_{k} \bar{a}_{k-2 n} \xi_{k}
$$


(b) The 2D-dimensional subspace $\mathcal{L}$ spanned by $\left\{e_{k} \mid-2 D+1 \leq k \leq 0\right\}$ is invariant under $S^{*}$, and the corresponding $2 D$ by $2 D$ matrix is

$$
\left(\begin{array}{cccccccc}
\bar{a}_{0} & 0 & 0 & \cdots & 0 & 0 & 0 & 0 \\
\bar{a}_{2} & \bar{a}_{1} & \bar{a}_{0} & \cdots & \vdots & \vdots & \vdots & \vdots \\
\vdots & \bar{a}_{3} & \bar{a}_{2} & \cdots & \vdots & & & \\
\bar{a}_{2 D-2} & \vdots & \vdots & & 0 & \vdots & \vdots & \vdots \\
0 & \bar{a}_{2 D-1} & \bar{a}_{2 D-2} & \ddots & \bar{a}_{0} & 0 & 0 & 0 \\
0 & 0 & 0 & & \bar{a}_{2} & \bar{a}_{1} & \bar{a}_{0} & 0 \\
\vdots & \vdots & \vdots & & \vdots & \bar{a}_{3} & \bar{a}_{2} & \bar{a}_{1} \\
\vdots & \vdots & \vdots & \cdots & \bar{a}_{2 D-2} & \vdots & \vdots & \vdots \\
0 & 0 & 0 & \cdots & 0 & \bar{a}_{2 D-1} & \bar{a}_{2 D-2} & \bar{a}_{2 D-3} \\
0 & 0 & 0 & \cdots & 0 & 0 & 0 & \bar{a}_{2 D-1}
\end{array}\right) .
$$

Specifically, the case $D=2$ is

$$
\left(\begin{array}{cccc}
\bar{a}_{0} & 0 & 0 & 0 \\
\bar{a}_{2} & \bar{a}_{1} & \bar{a}_{0} & 0 \\
0 & \bar{a}_{3} & \bar{a}_{2} & \bar{a}_{1} \\
0 & 0 & 0 & \bar{a}_{3}
\end{array}\right)
$$

and the space $\mathcal{L}$ is then spanned by $\left\{1, z^{-1}, z^{-2}, z^{-3}\right\}$.

(c) For all $n \in \mathbb{Z}$, there is a $k \in \mathbb{N}$ such that

$$
S^{* k} e_{n} \in \mathcal{L} \text {. }
$$

Proof. The matrix representations (2.25), and (2.26), follow directly from the formulas (2.23) and (2.16). To prove (b), set

$$
J(D):=\{k \in \mathbb{Z} \mid-2 D+1 \leq k \leq 0\},
$$

so that

$$
\mathcal{L}=\operatorname{span}\left\{e_{k} \mid k \in J(D)\right\} .
$$

We claim the following implication:

$$
\xi_{k}=0 \text { for } k \in \mathbb{Z} \backslash J(D) \Longrightarrow \sum_{k} \bar{a}_{k-2 n} \xi_{k}=0 \text { for } n \in \mathbb{Z} \backslash J(D) .
$$

The invariance of $\mathcal{L}$ under $S^{*}$ is immediate from this. Now suppose $\xi_{k}=0$ for $k \in \mathbb{Z} \backslash J(D)$. Then one of the factors in $\bar{a}_{k-2 n} \xi_{k}$ vanishes if $n \in \mathbb{Z} \backslash J(D)$; and the implication (2.30) follows. To understand (c), iterate the matrix formula (2.26). We get

$$
S^{*^{k}} e_{n}=\sum_{p}\left(\sum_{i_{1}, i_{2}, \cdots, i_{k}} \bar{a}_{i_{1}} \bar{a}_{i_{2}} \cdots \bar{a}_{i_{k}}\right) e_{p}
$$


where the summation on the right-hand side is over $p \in \mathbb{Z}, i_{1}, i_{2}, \cdots \in[0,1, \cdots, 2 D-$ 1], subject to

$$
i_{1}+2 i_{2}+\cdots+2^{k-1} i_{k}+p 2^{k}=n
$$

so the range for the $p$-index is roughly divided by 2 with each iteration of $S^{*}$ on the basis vector $e_{n}(z)=z^{n}$.

REMark 2.5. Conclusions (a)-(b) in Lemma 2.4 hold for the smaller subspace $\mathcal{M}:=\operatorname{span}\left\{e_{k} \mid-2 D-2 \leq k \leq 0\right\}$. The matrix of the restricted operator $\left.S^{*}\right|_{\mathcal{M}}$ is then obtained from (2.27) by deletion of the last row and last column. For example, the reduced matrix corresponding to (2.28) is

$$
F:=\left(\begin{array}{ccc}
\bar{a}_{0} & 0 & 0 \\
\bar{a}_{2} & \bar{a}_{1} & \bar{a}_{0} \\
0 & \bar{a}_{3} & \bar{a}_{2}
\end{array}\right) .
$$

However, property (c) in Lemma 2.4 is not satisfied for $\mathcal{M}$. To see this, note that $D=2$, and $\mathcal{L}=\mathcal{M} \oplus \mathbb{C} e_{-3}$ in the example. As a result

$$
\left\langle e_{-3} \mid S^{*^{k}} e_{-3}\right\rangle=\left(\bar{a}_{3}\right)^{k} \text { for all } k \in \mathbb{N} \text {. }
$$

If $a_{3} \neq 0$, it follows that $S^{*^{k}} e_{-3}$ is not in $\mathcal{M}$, no matter how large $k$ is.

\section{Measures Induced by Representations of the Cuntz Relations}

The system of coefficients studied in section 2 give rise to representations of the Cuntz relations. We recall the construction briefly; see [3] for more details. When the coefficients $a_{0}, a_{1}, \cdots$ are given, satisfying (2.3), then the two operators $S_{0}$ and $S_{1}$ from Lemma 2.1 (b) satisfy the relations (2.7). We say that the two operators satisfy the Cuntz relations, or equivalently that they define a representation of the Cuntz algebra $\mathcal{O}_{2}$ on the Hilbert space $\mathcal{H}=L^{2}(\mathbb{T})$. The norm-closed algebra of operators on $\mathcal{H}$ generated by $S_{0}, S_{1}, S_{0}^{*}$, and $S_{1}^{*}$ is known, [6] to be a simple $C^{*}$-algebra; and it is unique up to isomorphism of $C^{*}$-algebras.

We will also need to Hilbert space $L^{2}(\mathbb{R})$. If in addition to (2.3), the numbers $\left(a_{k}\right)$ satisfy

$$
\sum_{k=0}^{2 D-1} a_{k}=\sqrt{2}
$$

then it is known [5] that the following system of three equations has solutions in $L^{2}(\mathbb{R})$ :

$$
\begin{gathered}
\varphi_{2 n}(x)=\sqrt{2} \sum_{k} a_{k} \varphi_{n}(2 x-k), \\
\varphi_{2 n+1}(x)=\sqrt{2} \sum_{k}(-1)^{k} \overline{a_{2 D-1-k}} \varphi_{n}(2 x-k),
\end{gathered}
$$

and

$$
\int_{\mathbb{R}} \varphi_{0}(x) d x=1
$$


Moreover, for every $f \in L^{2}(\mathbb{R})$, the following Parseval identity holds:

$$
\sum_{n=0}^{\infty} \sum_{k \in \mathbb{Z}}\left|\left\langle\varphi_{n}(\cdot-k) \mid f\right\rangle\right|^{2}=\|f\|^{2}=\int_{\mathbb{R}}|f(x)|^{2} d x .
$$

When a system, such as

$$
\left\{\varphi_{n}(\cdot-k) \mid n \in \mathbb{N}_{0}, k \in \mathbb{Z}\right\},
$$

satisfies (3.5) we way it is a Parseval frame, or a normalized tight frame. There are simple further conditions on the coefficients $\left(a_{k}\right)$ each of which guarantees that (3.6) is in fact an orthonormal basis (ONB) in $L^{2}(\mathbb{R})$, i.e., that

$$
\left\langle\varphi_{n}(\cdot-k) \mid \varphi_{m}(\cdot-\ell)\right\rangle=\delta_{n, m} \delta_{k, \ell} .
$$

To understand how to select subsets $J \subset \mathbb{N}_{0} \times \mathbb{Z}$ such that the set of functions

$$
\mathcal{F}(J):=\left\{2^{\frac{j}{2}} \varphi_{n}\left(2^{j} x-k\right) \mid(n, j) \in J, k \in \mathbb{Z}\right\}
$$

forms a Perseval frame, or an ONB, for $L^{2}(\mathbb{R})$, the authors of [5] and [16] suggested a family of measures associated with the operator systems (2.7). We proved more generally, in 14 that these measures are associated with any representation of one of the Cuntz algebras $\mathcal{O}_{N}, N=2,3, \cdots$.

The idea is simple: Let $\left(S_{i}\right)_{i=0}^{1}$ be two operators in a Hilbert space $\mathcal{H}$ such that

$$
S_{i}^{*} S_{j}=\delta_{i, j} I \text { and } \sum_{i=0}^{1} S_{i} S_{i}^{*}=I
$$

hold. Then for every multi-index

$$
\xi:\left(i_{1}, i_{2}, \cdots, i_{k}\right), i_{j} \in\{0,1\}
$$

the operator

$$
P(\xi):=S_{i_{1}} \cdots S_{i_{k}} S_{i_{k}}^{*} \cdots S_{i_{1}}^{*}
$$

satisfies

$$
P(\xi)^{*}=P(\xi)=P(\xi)^{2} .
$$

Moreover

$$
\sum_{\xi} P(\xi)=I
$$

and

$$
P(\xi) P(\eta)=\delta_{\xi, \eta} P(\xi)
$$

where the summation in (3.12) is over all multi-indices of length $k$, and in (3.13) the multi-indices $\xi$ and $\eta$ have the same length. We say that, for each $k \in \mathbb{N}$ we have a dyadic partition of the Hilbert space $\mathcal{H}$ into orthogonal "frequency bands." 
Theorem 3.1. Let $m_{0}: \mathbb{T} \longrightarrow \mathbb{C}$ be a function which satisfies $m_{0}(1)=\sqrt{2}$ and

$$
\left|m_{0}(z)\right|^{2}+\left|m_{0}(-z)\right|^{2}=2 \quad \text { for } z \in \mathbb{T} \text {. }
$$

Set

$$
m_{1}(z)=z \overline{m_{0}(-z)}, \text { for } z \in \mathbb{T} ;
$$

and let $S_{i}, i=0,1$, be the operators (2.6). Suppose the sequence $\varphi_{0}, \varphi_{1}, \varphi_{2}, \cdots$ defined by

$$
\begin{gathered}
\widehat{\varphi_{2 n}}(x)=\frac{1}{\sqrt{2}} m_{0}\left(\frac{x}{2}\right) \widehat{\varphi_{n}}\left(\frac{x}{2}\right) \\
\widehat{\varphi_{2 n+1}}(x)=\frac{1}{\sqrt{2}} m_{1}\left(\frac{x}{2}\right) \widehat{\varphi_{n}}\left(\frac{x}{2}\right)
\end{gathered}
$$

for $n=0,1, \cdots, x \in \mathbb{R}$ defines an orthonormal basis in $L^{2}(\mathbb{R})$, where we set $m_{j}(x):=m_{j}\left(e^{i 2 \pi x}\right), j=0,1$. Then

$2^{\frac{p}{2}} \varphi_{n}\left(2^{p} t-k\right)=\sum_{i_{1}=0}^{1} \cdots \sum_{i_{p}=0}^{1} \sum_{j \in \mathbb{Z}}\left\langle e_{j} \mid S_{i_{p}}^{*} \cdots S_{i_{1}}^{*} e_{k}\right\rangle \varphi_{2^{p} n+i_{1}+i_{2} 2+\cdots+i_{p} 2^{p-1}}(t-j)$

holds for all $p, n \in \mathbb{N}_{0}$ and $k \in \mathbb{Z}$. Moreover a subset $J \subset \mathbb{N}_{0} \times \mathbb{N}_{0}$ has the corresponding set

$$
\left\{2^{\frac{p}{2}} \varphi_{n}\left(2^{p} t-k\right) \mid(p, n) \in J, k \in \mathbb{Z}\right\}
$$

define an orthonormal basis (ONB) in $L^{2}(\mathbb{R})$ if and only if the sets $\left[2^{p} n, 2^{p}(n+1)\right)$ form a non-overlapping partition of $\mathbb{N}_{0}$.

Proof. Since the functions in (3.16)-3.17) form an orthonormal basis for $L^{2}(\mathbb{R})$, we have

$$
\sum_{j \in \mathbb{Z}} \widehat{\widehat{\varphi_{n}}(x+j)} \widehat{\varphi_{n^{\prime}}}(x+j)=\delta_{n, n^{\prime}} \text { for } n, n^{\prime} \in \mathbb{N}_{0},
$$

and (3.18) will follow if we check that

$$
\int_{\mathbb{R}} \overline{\varphi_{m}(t-j)} 2^{\frac{p}{2}} \varphi_{n}\left(2^{p} t-k\right) d t=\left\langle e_{j} \mid S_{i_{p}}^{*} \cdots S_{i_{1}}^{*} e_{k}\right\rangle
$$

if $m=2^{p} n+i_{1}+i_{2} 2+\cdots+i_{p} 2^{p-1}$, and zero otherwise. But (3.21) follows from Fourier duality, and a substitution of formulas (3.16)-(3.17) and (3.20). We leave the computation to the reader. The second conclusion regarding the basis properties (ONB) of the functions in (3.19) follows from (3.18) when we note that

$$
m=2^{p} n+i_{1}+i_{2} 2+\cdots+i_{p} 2^{p-1}
$$

ranges over $\left[2^{p} n, 2^{p}(n+1)\right)$ as $i_{1}, \cdots, i_{p}$ vary over $\underbrace{\mathbb{Z}_{2} \times \cdots \times \mathbb{Z}_{2}}_{p \text { times }}$. 
EXAMPLE 3.2. The best known special case of 3.19) is when

$$
J=\{(0,1),(1,1),(2,1),(3,1), \cdots\}
$$

and the corresponding partition of $\mathbb{N}_{0}$ is simply $\left[2^{p}, 2^{p+1}\right)$ for $p=0,1,2, \cdots$. In this case, we only need the first two functions from the sequence $\varphi_{n}$ of (3.16)(3.17), i.e., $\varphi_{0}=\varphi$, and $\varphi_{1}=\psi$. The other extreme is $J=\{(0,1),(0,2),(0,3)$, $\cdots\}$. In this case, each section in the corresponding partition of $\mathbb{N}_{0}$ is a singleton, and all the functions $\left\{\varphi_{n} \mid n \in \mathbb{N}_{0}\right\}$ from (3.16)-(3.17) are needed in the (3.19) family. However, no dyadic scales are used. Between these two extremes there is a countable infinite family of non-overlapping partitions corresponding to the choice specified in (3.19); for example, the pairs $(p, n)$ in some $J$ from (3.19) may be specified as in the following table (Table 1).

\section{Table 1.}

A set $J$ consisting of points $(p, n)$ as follows:

$\begin{array}{lllllllllllllll}p & 2 & 2 & 2 & 2 & 4 & 4 & 4 & 6 & 6 & 6 & 8 & 8 & 8 & \ldots \\ n & 0 & 1 & 2 & 3 & 1 & 2 & 3 & 1 & 2 & 3 & 1 & 2 & 3 & \ldots\end{array}$

In this case, we are using only the four functions $\varphi_{0}, \varphi_{1}, \varphi_{2}$, and $\varphi_{3}$ from (3.16) (3.17), and the segments from (3.19) are $\{0,1,2,3\}$ followed by

$$
\left[2^{2 k} j, 2^{2 k}(j+1)\right)
$$

where $k=1,2,3, \cdots$, and $j \in\{1,2,3\}$. As a result, all the dyadic scaling numbers $2^{2 k}$ are used. The resulting ONB in (3.19) consists of the following functions, $2 \varphi_{0}\left(2^{2} t-\ell_{0}\right), 2^{k} \varphi_{j}\left(2^{2 k} t-\ell\right)$, where $\ell_{0}, \ell \in \mathbb{Z}, k=1,2, \cdots$, and $j \in\{1,2,3\}$.

Since $\left\{\varphi_{m}(\cdot-j) \mid m \in \mathbb{N}_{0}, j \in \mathbb{Z}\right\}$ is an ONB for $L^{2}(\mathbb{R})$, every $f \in L^{2}(\mathbb{R})$, $\|f\|^{2}=1$, defines a probability distribution $P_{f}$ on $\mathbb{N}_{0} \times \mathbb{Z}$ by

$$
P_{f}(m, j):=\left|\left\langle\varphi_{m}(\cdot-j) \mid f\right\rangle\right|^{2}
$$

Corollary 3.3. Let $p, n \in \mathbb{N}_{0}, k \in \mathbb{Z}$, and let $P_{2^{\frac{p}{2}} \varphi_{n}\left(2^{p \cdot-k)}\right.}(\cdot, \cdot)$ be the corresponding probability distribution from (3.22) above. Then the marginal distribution in the first variable is

$$
\delta_{m, 2^{p} n+i_{1}+i_{2} 2+\cdots+i_{p} 2^{p-1}} \mu_{e_{k}}\left(\left[\frac{i_{1}}{2}+\cdots+\frac{i_{p}}{2^{p}}, \frac{i_{1}}{2}+\cdots+\frac{i_{p}}{2^{p}}+\frac{1}{2^{p}}\right)\right) .
$$


Proof. In view of (3.22) and Theorem 3.1 the marginal distribution is

$$
\begin{aligned}
& \sum_{j \in \mathbb{Z}} P_{2^{\frac{p}{2}} \varphi_{n}\left(2^{p}-k\right)}(m, j) \\
& =\delta_{m, 2^{p} n+i_{1}+i_{2} 2+\cdots+i_{p} 2^{p-1}} \sum_{j \in \mathbb{Z}}\left|\left\langle e_{j} \mid S_{i_{p}}^{*} \cdots S_{i_{1}}^{*} e_{k}\right\rangle\right|^{2} \\
& =\delta_{m, 2^{p} n+i_{1}+i_{2} 2+\cdots+i_{p} 2^{p-1}}\left\|S_{i_{p}}^{*} \cdots S_{i_{1}}^{*} e_{k}\right\|^{2} \\
& =\delta_{m, 2^{p} n+i_{1}+i_{2} 2+\cdots+i_{p} 2^{p-1}} \mu_{e_{k}}\left(\left[\frac{i_{1}}{2}+\cdots+\frac{i_{p}}{2^{p}}, \frac{i_{1}}{2}+\cdots+\frac{i_{p}}{2^{p}}+\frac{1}{2^{p}}\right)\right)
\end{aligned}
$$

which is the desired conclusion.

The next result follows from [5], or from [14.

Lemma 3.4. There is a unique Borel measure $E$ defined on the unit interval $[0,1]$ such that

$$
E\left(\left[\frac{i_{1}}{2}+\cdots+\frac{i_{k}}{2^{k}}, \frac{i_{1}}{2}+\cdots+\frac{i_{k}}{2^{k}}+\frac{1}{2^{k}}\right)\right)=P\left(i_{1}, i_{2}, \cdots, i_{k}\right)=S_{\xi} S_{\xi}^{*}
$$

Moreover the measure $E=E_{P}$ takes values in orthogonal projections in $\mathcal{H}$, and

$$
\begin{gathered}
\int_{0}^{1} E(d x)=I \\
E\left(B_{1}\right) E\left(B_{2}\right)=0 \text { if } B_{i} \text { are Borel sets with } B_{1} \bigcap B_{2}=\varnothing .
\end{gathered}
$$

REMARK 3.5. The expectation is that the measure class of $E$ is that of the Lebesgue measure if $E$ is derived from a wavelet representation. For the wavelet representations, the Hilbert space $\mathcal{H}$ is $L^{2}(\mathbb{T})$; and for a generic set of wavelet representations $e_{0}$ is a cyclic vector; see 13. Hence, to check if $E$ is in the Lebesgue class, it is enough if we verify that the scalar valued, positive measure

$$
\mu_{0}(\cdot):=\left\langle e_{0} \mid E(\cdot) e_{0}\right\rangle=\left\|E(\cdot) e_{0}\right\|^{2}
$$

is absolutely continuous with respect to Lebesgue measure on [0,1]. Using (3.24), we see that the measure $\mu_{0}$ is given on dyadic intervals by the formula

$$
\mu_{0}\left(\left[\frac{i_{1}}{2}+\cdots+\frac{i_{k}}{2^{k}}, \frac{i_{1}}{2}+\cdots+\frac{i_{k}}{2^{k}}+\frac{1}{2^{k}}\right)\right)=\left\|S_{\xi}^{*} e_{0}\right\|^{2} .
$$

Part of the reason for expecting that the measure $\mu_{0}(\cdot)=\left\|E(\cdot) e_{0}\right\|^{2}$ should be absolutely continuous with respect to Lebesgue measure on $[0,1]$ rests on the observation that $\lambda=\frac{1}{\sqrt{2}}$ is always in the spectrum of the matrix (2.27). If 
$\lambda=\frac{1}{\sqrt{2}}$ is also a dominant eigenvalue, in a sense which we make precise below, then the asymptotic formula

$$
\mu_{0}\left(\left[\frac{i_{1}}{2}+\cdots+\frac{i_{k}}{2^{k}}, \frac{i_{1}}{2}+\cdots+\frac{1}{2^{k}}+\frac{1}{2^{k}}\right)\right) \cong 2^{-k}
$$

would follow. From this the absolute continuity follows as well. But a closer examination of the matrices $F_{i}, i=0,1$, shows that $\lambda=\frac{1}{\sqrt{2}}$ is not always a dominant point in the spectrum of $F_{0}=\left.S_{0}^{*}\right|_{\mathcal{M}}$.

We now show that $\frac{1}{\sqrt{2}}$ is in the spectrum.

LEMma 3.6. Let the numbers $a_{0}, a_{1}, \cdots, a_{2 D-1}$ satisfy (2.3) and (3.1), and let $S_{0}$ and $S_{0}^{*}$ be the corresponding operators on $\ell^{2}$; see 2.25) and (2.26). Then $\lambda=\frac{1}{\sqrt{2}}$ is an eigenvalue for the finite-dimensional operator $F_{0}=\left.S_{0}^{*}\right|_{\mathcal{M}}$. If $F_{0}$ is represented as a $2 D-1$ by $2 D-1$ matrix as in (2.27), then the rowvector $w=\underbrace{(1,1, \cdots, 1)}_{2 D-1}$ is a left-eigenvector for $F_{0}$, or equivalently $w^{*}$ is a right-eigenvector, with the same eigenvalue $\frac{1}{\sqrt{2}}$, for the adjoint matrix $F_{0}^{*}$.

Proof. Substitution of $m_{0}(1)=\sqrt{2}$ into $\left|m_{0}(z)\right|^{2}+\left|m_{0}(-z)\right|^{2}=2$ from Corollary 2.3 yields $m_{0}(-1)=0$. Hence, $z+1$ divides the polynomial $m_{0}(z)$. If

$$
\begin{aligned}
m_{0}(z) & =(z+1) \mathcal{L}(z), \text { and } \\
\mathcal{L}(z) & =\sum_{i=0}^{2 D-2} \ell_{i} z^{i}, \text { then it follows that } \\
\mathcal{L}(1) & =\sum_{i=0}^{2 D-2} \ell_{i}=\frac{\sqrt{2}}{2}=\frac{1}{\sqrt{2}} .
\end{aligned}
$$

As a result we get

$$
\left\{\begin{array}{l}
a_{0}=\ell_{0} \\
a_{i}=\ell_{i}+\ell_{i-1} \text { for } 1 \leq i \leq 2 D-2 \\
a_{2 D-1}=\ell_{2 D-2} .
\end{array}\right.
$$

It follows from this that

$$
\sum_{i=0}^{D-1} a_{2 i}=\sum_{i} \ell_{i}=\frac{1}{\sqrt{2}}
$$

and

$$
\sum_{i=0}^{D-1} a_{2 i+1}=\sum_{i} \ell_{i}=\frac{1}{\sqrt{2}}
$$

Working out the matrix product, this is a restatement of $w F_{0}=\frac{1}{\sqrt{2}} w$, or equivalently $F_{0}^{*} w^{*}=\frac{1}{\sqrt{2}} w^{*}$. 
THEOREM 3.7. Let $a_{0}, a_{1}, \cdots a_{2 D-1}$ be as stated in Lemma 3.6 suppose

$$
\left|a_{0}\right|>\max \left\{|\lambda| \mid \lambda \in \operatorname{spec}\left(F_{0}\right) \backslash\left\{a_{0}\right\}\right\},
$$

and set

$$
s=-\frac{\ln \left|a_{0}\right|^{2}}{\ln 2} .
$$

Then every non-empty open subset $V$ in $[0,1]$ contains an infinite sequence of dyadic intervals $J$ such that

$$
0<\liminf _{J \subset V}\left(\frac{\mu_{0}(J)}{|J|^{s}}\right) \leq \limsup _{J \subset V}\left(\frac{\mu_{0}(J)}{|J|^{s}}\right)<\infty .
$$

We say that the measure $\mu_{0}$ contains $s$ as a fractal scale.

Proof. The details follow by combining Lemma [3.6] with the technical lemma which we state and prove in Section 5 below.

Proposition 3.8. Let the numbers $a_{0}, \cdots, a_{2 D-1}$ satisfy the two conditions (2.3) and (3.1) and let $S_{0}, S_{1}$ be the corresponding two operators; see (2.6)-(2.7). Let $\mu_{0}(\cdot):=\left\|E(\cdot) e_{0}\right\|^{2}$ be the measure (3.28). Then

$$
\mu_{0}\left(\left[\frac{i_{1}}{2}+\cdots+\frac{i_{k}}{2^{k}}, \frac{i_{1}}{2}+\cdots+\frac{i_{k}}{2^{k}}+\frac{1}{2^{k}}\right)\right) \geq\left|a_{0}\right|^{2 \cdot \#(i=0)}\left|a_{2 D-1}\right|^{2 \cdot \#(i=1)} .
$$

REMARK 3.9. We will see later how this estimate yields information about a fractal component of $\mu_{0}$ in the special case $D=2$.

Proof. Let $F_{i}:=\left.S_{i}^{*}\right|_{\mathcal{M}}, i=0,1$. For $k \in \mathbb{N}$, and $i_{1}, \cdots, i_{k} \in\{0,1\}$, set $\xi=\frac{i_{1}}{2}+\cdots+\frac{i_{k}}{2^{k}}$ and $F_{\xi}:=F_{i_{k}} \cdots F_{i_{1}}$. Then $F_{0}^{*} e_{0}=\bar{a}_{0} e_{0}$ and $F_{1}^{*} e_{0}=\bar{a}_{2 D-1} e_{0}$. To see this, recall that

$$
m_{1}(z)=\bar{a}_{2 D-1}-\bar{a}_{2 D-2} z+\cdots-\bar{a}_{0} z^{2 D-1}
$$

and

$$
\begin{aligned}
\mu_{0}\left(\left[\xi, \xi+2^{-k}\right)\right) & =|| F_{\xi} e_{0} \|^{2} \geq\left|\left\langle e_{0} \mid F_{\xi} e_{0}\right\rangle\right|^{2} \\
& =\left|\left\langle F_{i_{1}}^{*} \cdots F_{i_{k}}^{*} e_{0} \mid e_{0}\right\rangle\right|^{2} \\
& =\left|a_{0}\right|^{2 \cdot \#(i=0)}\left|a_{2 D-1}\right|^{2 \cdot \#(i=1)}
\end{aligned}
$$

LEMma 3.10. (The case $D=2$.)

(a) For the case $D=2$, the real valued solutions $a_{0}, a_{1}, a_{2}, a_{3}$ to the three equations

$$
\left\{\begin{array}{l}
a_{0}^{2}+a_{1}^{2}+a_{2}^{2}+a_{3}^{2}=1 \\
a_{0} a_{2}+a_{1} a_{3}=0 \\
a_{0}+a_{1}+a_{2}+a_{3}=\sqrt{2}
\end{array}\right.
$$


are

$$
\left\{\begin{array}{l}
a_{0}=\frac{1}{2 \sqrt{2}}(1+\sqrt{2} \cos \beta) \\
a_{1}=\frac{1}{2 \sqrt{2}}(1+\sqrt{2} \sin \beta) \\
a_{2}=\frac{1}{2 \sqrt{2}}(1-\sqrt{2} \cos \beta) \\
a_{3}=\frac{1}{2 \sqrt{2}}(1-\sqrt{2} \sin \beta)
\end{array}\right.
$$

for $\beta \in \mathbb{R}$.

(b) The spectrum of the matrix

$$
F=\left(\begin{array}{ccc}
a_{0} & 0 & 0 \\
a_{2} & a_{1} & a_{0} \\
0 & a_{3} & a_{2}
\end{array}\right)
$$

obtained by restriction of $S^{*}$ to $\mathcal{M}=\operatorname{span}\left\{e_{0}, e_{-1}, e_{-2}\right\}$ is

$$
\left\{a_{0}, \frac{1}{\sqrt{2}}, \frac{\sin \beta-\cos \beta}{2}\right\} \text {. }
$$

(c) The following two conditions are equivalent

$$
a_{0}>\frac{1}{\sqrt{2}} \geq \max \left\{|\lambda| \mid \lambda \in \operatorname{spec}(F) \backslash\left\{a_{0}\right\}\right\}
$$

and

$$
|\beta|<\frac{\pi}{4} .
$$

(d) The eigenspace for the point $a_{0}$ in the spectrum of $F$ is spanned by the vector $\left(\begin{array}{l}1 \\ v\end{array}\right)$ where $v \in \mathbb{R}^{2}$ is the solution to

$$
v=\left(a_{0} I_{2}-\left(\begin{array}{cc}
a_{1} & a_{0} \\
a_{3} & a_{2}
\end{array}\right)\right)^{-1}\left(\begin{array}{c}
a_{2} \\
0
\end{array}\right) .
$$

(e) For the case of Daubechies's wavelet, we have

$$
\begin{gathered}
\left\{\begin{array}{l}
a_{0}=\frac{1+\sqrt{3}}{4 \sqrt{2}} \\
a_{1}=\frac{3+\sqrt{3}}{4 \sqrt{2}} \\
a_{2}=\frac{3-\sqrt{3}}{4 \sqrt{2}}, \\
a_{3}=\frac{1-\sqrt{3}}{4 \sqrt{2}}
\end{array}\right. \\
\beta=\arccos \left(\frac{\sqrt{3}-1}{2 \sqrt{2}}\right),
\end{gathered}
$$

and

$$
\operatorname{spec}(F)=\left\{a_{0}, \frac{1}{\sqrt{2}}, \frac{1}{2 \sqrt{2}}\right\} .
$$

In particular,

$$
a_{0}<\frac{1}{\sqrt{2}}=\max \{|\lambda| \mid \lambda \in \operatorname{spec}(F)\} .
$$


Proof. Part (a) follows directly from section 4 in [2]. To compute the spectrum of the matrix $F$, note that its characteristic polynomial is

$$
\left(\lambda-a_{0}\right)\left(\lambda^{2}-\left(a_{1}+a_{2}\right) \lambda+a_{1} a_{2}-a_{0} a_{3}\right) ;
$$

and when (3.33) are substituted, we see that the roots are as listed in (3.35). The conclusions listed in parts (c) and (d) are immediate consequences of (b). Finally (3.38) follows from the observation that $F$ has the form

$$
F=\left(\begin{array}{ccc}
a_{0} & 0 & 0 \\
a_{2} & & \\
0 & &
\end{array}\right)
$$

with $G=\left(\begin{array}{cc}a_{1} & a_{0} \\ a_{3} & a_{2}\end{array}\right)$ and the fact that $a_{0}$ is not in the spectrum of $G$. The conclusions listed in (e) for Daubechies's wavelet follow by an application of (a) $-(d)$.

To better appreciate the geometry of the formulas (3.33), the reader may find Fig. 1 useful.

The coefficients $a_{0}(\beta), a_{1}(\beta), a_{2}(\beta), a_{3}(\beta)$ of (3.32)

\section{Figure 1.}

The four coefficients $a_{0}, a_{1}, a_{2}, a_{3}$ are described by the two circles in Fig. 1, viz., by

$\left(a_{0}-\frac{1}{2 \sqrt{2}}\right)^{2}+\left(a_{3}-\frac{1}{2 \sqrt{2}}\right)^{2}=\frac{1}{4}$, and by $\left(a_{1}-\frac{1}{2 \sqrt{2}}\right)^{2}+\left(a_{2}-\frac{1}{2 \sqrt{2}}\right)^{2}=\frac{1}{4}$.

This representation also makes it clear that, if one of the four coefficients vanishes, then so does a second from the remaining coefficients. The resulting four degenerate cases are as sketched in Table 2 . 


\section{Table 2.}

The degenerate cases when one (and therefore two) of the coefficients $a_{0}, a_{1}, a_{2}, a_{3}$ vanish:

$$
\begin{array}{ccccc}
m_{0} & \frac{1+z}{\sqrt{2}} & \frac{1+z^{3}}{\sqrt{2}} & \frac{z+z^{2}}{\sqrt{2}} & \frac{z^{2}+z^{3}}{\sqrt{2}} \\
m_{1} & \frac{z^{2}-z^{3}}{\sqrt{2}} & \frac{1-z^{3}}{\sqrt{2}} & \frac{-z+z^{2}}{\sqrt{2}} & \frac{1-z}{\sqrt{2}} \\
\beta & \frac{\pi}{4} & -\frac{\pi}{4} & \frac{3 \pi}{4} & -\frac{3 \pi}{4} \\
\hline
\end{array}
$$

There are four distinct configurations, and all four are variations of the Haar wavelet, see Fig. 2. The second column in Table 2 is called the stretched Haar wavelet; see [3]. For all three, we have the formula

$$
\mu_{0}\left(\left[\frac{i_{1}}{2}+\cdots+\frac{i_{k}}{2^{k}}, \frac{i_{1}}{2}+\cdots+\frac{i_{k}}{2^{k}}+\frac{1}{2^{k}}\right)\right)=2^{-k}
$$

which proves that $\mu_{0}$ is the Lebesgue measure on the unit-interval $[0,1]$ for all four variations of the Haar wavelet. The two matrices $F_{0}$ and $F_{1}$ corresponding to the four variants of the Haar wavelet filters in Table 2 are as follows:

$$
\begin{aligned}
& F_{0}: \frac{1}{\sqrt{2}}\left(\begin{array}{ccc}
1 & 0 & 0 \\
0 & 1 & 1 \\
0 & 0 & 0
\end{array}\right) \frac{1}{\sqrt{2}}\left(\begin{array}{ccc}
1 & 0 & 0 \\
0 & 0 & 1 \\
0 & 1 & 0
\end{array}\right) \frac{1}{\sqrt{2}}\left(\begin{array}{ccc}
0 & 0 & 0 \\
1 & 1 & 0 \\
0 & 0 & 1
\end{array}\right) \frac{1}{\sqrt{2}}\left(\begin{array}{ccc}
0 & 0 & 0 \\
1 & 0 & 0 \\
0 & 1 & 1
\end{array}\right) \\
& F_{1}: \frac{1}{\sqrt{2}}\left(\begin{array}{ccc}
0 & 0 & 0 \\
1 & 0 & 0 \\
0 & -1 & 1
\end{array}\right) \frac{1}{\sqrt{2}}\left(\begin{array}{ccc}
1 & 0 & 0 \\
0 & 0 & 1 \\
0 & -1 & 0
\end{array}\right) \frac{1}{\sqrt{2}}\left(\begin{array}{ccc}
0 & 0 & 0 \\
1 & -1 & 0 \\
0 & 0 & 1
\end{array}\right) \frac{1}{\sqrt{2}}\left(\begin{array}{ccc}
1 & 0 & 0 \\
0 & -1 & 1 \\
0 & 0 & 0
\end{array}\right)
\end{aligned}
$$


The father function $\varphi$ and the mother function $\psi$ for the Haar Wavelet

Figure 2.

REMARK 3.11. We shall need Lemma 3.6 for the two matrices

$$
F_{0}=\left(\begin{array}{ccc}
a_{0} & 0 & 0 \\
a_{2} & a_{1} & a_{0} \\
0 & a_{3} & a_{2}
\end{array}\right) \text { and } F_{1}=\left(\begin{array}{ccc}
a_{3} & 0 & 0 \\
a_{1} & -a_{2} & a_{3} \\
0 & -a_{0} & a_{3}
\end{array}\right)
$$

For the range $|\beta|<\frac{\pi}{4}$, we have (3.36) satisfied by spec $\left(F_{0}\right)$. But then the point $a_{3}=a_{3}(\beta)$ satisfies $\left|a_{3}\right|<\frac{1}{\sqrt{2}}$, and

$$
\frac{1}{\sqrt{2}}=\max \left\{|\lambda| \mid \lambda \in \operatorname{spec}\left(F_{1}\right)\right\} .
$$

Theorem 3.12. Let $\beta \in \mathbb{R},|\beta|<\frac{\pi}{4}$, be given. Let $a_{0}, a_{1}, a_{2}, a_{3}$, be the corresponding numbers in (3.33). Let $\left(S_{i}\right)_{i=0}^{1}$ be the operators in (2.6), and

$$
F_{i}:=\left.S_{i}^{*}\right|_{\mathcal{M}}
$$

the matrices (3.47). Let

$$
\xi=\frac{i_{1}}{2}+\cdots+\frac{i_{k}}{2^{k}}
$$

be a dyadic fraction. Then

$$
\lim _{n \rightarrow \infty} a_{0}^{-n} F_{0}^{n} F_{i_{k}} \cdots F_{i_{1}} e_{0}=a_{0}^{\#(i=0)} a_{3}^{\#(i=1)}\left(e_{0}+v\right)
$$


where $v$ is the vector in (3.38). Moreover, if $\varepsilon>0$ is given, there is an $n_{0} \in \mathbb{N}$ such that

$$
\mu_{0}\left(\left[\xi, \xi+\frac{1}{2^{n+k}}\right)\right) \geq a_{0}^{2 n} a_{0}^{2 \cdot \#(i=0)} a_{3}^{2 \cdot \#(i=1)}\left(1+\|v\|^{2}-\varepsilon\right) .
$$

for all $n \geq n_{0}$. More generally, given $\varepsilon, 0<\varepsilon<1$, there is $n_{0} \in \mathbb{N}$ such that

$$
\begin{aligned}
\left(1+\|v\|^{2}-\varepsilon\right)\left(a_{0}^{\#(i=0)} a_{3}^{\#(i=1)}\right)^{2} & \leq \frac{\mu_{0}\left(\left[\xi, \xi+\frac{1}{2^{n+k}}\right)\right)}{a_{0}^{2 n}} \\
& \leq\left(a_{0}^{\#(i=0)} a_{3}^{\#(i=1)}\right)^{2}\left(1+\|v\|^{2}+\varepsilon\right) \text { for all } n \geq n_{0} .
\end{aligned}
$$

Proof. Recall that $e_{0}+v$ is the eigenvector satisfying

$$
F_{0}\left(e_{0}+v\right)=a_{0}\left(e_{0}+v\right) \text {, and }\left\langle e_{0} \mid e_{0}+v\right\rangle=1 .
$$

Moreover, we have

$$
F_{0}^{*} e_{0}=a_{0} e_{0} \text { and } F_{1}^{*} e_{0}=a_{3} e_{0} .
$$

Using (3.36) for $F_{0}$, we get

$$
\lim _{n \rightarrow \infty} a_{0}^{-n} F_{0}^{n} w=\left\langle e_{0} \mid w\right\rangle\left(e_{0}+v\right)
$$

for all $w \in \mathcal{M}$. Applying this, and (3.54), to $w:=F_{i_{k}} \cdots F_{i_{1}} e_{0}$, the conclusion (3.51) follows. Since

$$
\mu_{0}\left(\left[\xi, \xi+\frac{1}{2^{n+k}}\right)\right)=\left\|S_{0}^{*^{n}} S_{\xi}^{*} e_{0}\right\|^{2}=\left\|F_{0}^{n} F_{i_{k}} \cdots F_{i_{1}} e_{0}\right\|^{2},
$$

the second conclusion (3.52) follows from the first.

Corollary 3.13. Let $|\beta|<\frac{\pi}{4}$, and let $a_{0}, a_{1}, a_{2}, a_{3}$ be the corresponding numbers in (3.33). If the measure $\mu_{0}(\cdot)=\left\|E(\cdot) e_{0}\right\|^{2}$ is absolutely continuous with respect to Lebesgue measure $d x$ on $[0,1]$. Then the Radon-Nikodym derivative $d \mu_{0} / d x=f$ is unbounded in every open (non-empty) subset of $[0,1]$.

Proof. Let $V \subset[0,1]$ be a non-empty open subset. Then pick $\xi$ as in (3.50), and $n_{1} \in \mathbb{N}$ such that

$$
\left[\xi, \xi+\frac{1}{2^{n_{1}}}\right) \subset V .
$$

Then pick $\varepsilon, 0<\varepsilon<\frac{1}{2}$, and $n_{0} \geq n_{1}$ such that (3.52) holds for all $n \geq n_{0}$. If $f$ were bounded in $V$ with upper bound $C$; then (3.52) implies the estimate

$$
C \cdot 2^{-n} \geq a_{0}^{2 n} a_{0}^{2 \cdot \#(i=0)} a_{3}^{2 \cdot \#(i=1)}\left(1+\|v\|^{2}-\varepsilon\right)
$$

for all $n \geq n_{0}$. Since $a_{0}^{2}>\frac{1}{2}, \lim _{n \longrightarrow \infty} 2^{n} a_{0}^{2 n}=\infty$, which contradicts (3.58). 
EXAMPLE 3.14. We introduced the constants $a_{0}, a_{1}, a_{2}, a_{3}$ for Daubechies's wavelet in part (e) of Lemma 3.6 An inspection of (3.33) and (3.39) shows that $\beta=\arccos \left(\frac{\sqrt{3}-1}{2 \sqrt{2}}\right)$. Hence

$$
\frac{\sin \beta-\cos \beta}{2}=\frac{1}{2 \sqrt{2}}
$$

and the three numbers in the spectrum of $F_{0}$ are as follows:

$$
\frac{1}{2 \sqrt{2}}<a_{0}<\frac{1}{\sqrt{2}}
$$

One checks that the vector

$$
v^{*}:=(1+\sqrt{3}) e_{-1}+(1-\sqrt{3}) e_{-2}
$$

spans the $\frac{1}{\sqrt{2}}$-eigenspace for $F_{0}$. Since $a_{0}<\frac{1}{\sqrt{2}}$, the eigenspace

$$
\left\{w \in \mathcal{M} \mid F_{0} w=\frac{1}{\sqrt{2}} w\right\}
$$

is orthogonal to $e_{0}$.

Corollary 3.15. Let $\beta=\arccos \left(\frac{\sqrt{3}-1}{2 \sqrt{2}}\right)$, so that the numbers $a_{0}, a_{1}, a_{2}$, $a_{3}$ define the Daubechies wavelet. Let $v^{*}$ be the eigenvector in (3.60), and pick $w \in \mathcal{M}$ such that

$$
F_{0}^{*} w=\frac{1}{\sqrt{2}} w, \text { and }\left\langle w \mid v^{*}\right\rangle=\sqrt{8}
$$

Let $\xi$ be a dyadic rational given as in (3.50). Then, for every $\varepsilon, 0<\varepsilon<1$, there is $n_{0} \in \mathbb{N}$ such that

$$
\begin{aligned}
\left|\left\langle w \mid F_{i_{k}} \cdots F_{i_{1}} e_{0}\right\rangle\right|^{2}-\varepsilon & \leq \frac{\mu_{0}\left(\left[\xi, \xi+\frac{1}{2^{n+k}}\right)\right)}{2^{-n}} \\
& \leq\left|\left\langle w \mid F_{i_{k}} \cdots F_{i_{1}} e_{0}\right\rangle\right|^{2}+\varepsilon \text { for all } n \geq n_{0}
\end{aligned}
$$

Proof. The argument follows the proof of Theorem 3.12 The vector $w$ is $w=\sqrt{2}\left(e_{0}+e_{-1}+e_{-2}\right)$. The main difference is that now $\frac{1}{\sqrt{2}}$ is the top eigenvalue of $F_{0}$ with eigenvector $v^{*}$. If $w$ is chosen as in (3.62), then

$$
\lim _{n \longrightarrow \infty} 2^{\frac{n}{2}} F_{0}^{n} s=\frac{1}{\sqrt{8}}\langle w \mid s\rangle v^{*}
$$

for all $s \in \mathcal{M}$. The desired result (3.63) follows when this is applied to $s=$ $F_{i_{k}} \cdots F_{i_{1}} e_{0}$. 


\section{Wavelets on Fractals}

In section 2 we proved some lemmas for representations of the Cuntz algebra $\mathrm{O}_{2}$, and we used them in section 3 above in our analysis of dyadic wavelets in the Hilbert space $L^{2}(\mathbb{R})$. But there are other Hilbert spaces $\mathcal{H}$ which admit wavelet algorithms. In a recent paper [9], we constructed wavelets in separable Hilbert spaces $\mathcal{H}^{(s)}$ built on Hausdorff measure $(d x)^{s}$ where $s$ denotes the corresponding fractal dimension. For the middle third Cantor set, for example, the scaling number $N$ is 3 ; and the fractal dimension is $s=\frac{\ln 2}{\ln 3}=\log _{3}(2)$.

In this section, we show how our Cantor subdivision construction is built on a representation of the $\mathcal{O}_{3}$-Cuntz relations on $L^{2}(\mathbb{T})$ which are analogous to the representations of $\mathcal{O}_{2}$ which we used in our analysis of dyadic $L^{2}(\mathbb{R})$-wavelets.

Lemma 4.1. Let $N \in \mathbb{N}, N \geq 2$, be given, and let $m_{0}, m_{1}, \cdots, m_{N-1}$ be bounded measurable functions on $\mathbb{T}$. Then the operators

$$
\left(S_{i} f\right)(z)=m_{i}(z) f\left(z^{N}\right), f \in L^{2}(\mathbb{T}), i=0,1, \cdots, N-1, z \in \mathbb{T}
$$

satisfy the Cuntz relations

$$
\left\{\begin{array}{c}
S_{i}^{*} S_{j}=S_{i, j} I \\
\sum_{i=0}^{N-1} S_{i} S_{i}^{*}=I
\end{array}\right.
$$

if and only if the associated $N$ by $N$ matrix function

$$
M_{N}(z)=\frac{1}{\sqrt{N}}\left(m_{j}\left(z e^{i \frac{k 2 \pi}{N}}\right)\right)_{j, k}
$$

takes values in the unitary matrices for a.e. $z \in \mathbb{T}$.

Proof. Rather than sketching the details, we will instead refer the reader to section 2 above where the argument is done in full for $N=2$; see also [9] or [3].

For each of the representations $\left(S_{i}\right)_{i=0}^{N-1}$, we get a measure $\mu_{0}$ on the unitinterval $[0,1]$, as outlined in section 3 (for the special case $N=2$.) In the general case, the measure $\mu_{0}$ is determined uniquely on the $N$-adic subintervals as follows,

$$
\mu_{0}\left(\left[\frac{i_{1}}{N}+\cdots+\frac{i_{k}}{N^{k}}, \frac{i_{1}}{N}+\cdots+\frac{i_{k}}{N^{k}}+\frac{1}{N^{k}}\right)\right)=\left\|S_{i_{k}}^{*} \cdots S_{i_{2}}^{*} S_{i_{1}}^{*} e_{0}\right\|^{2}
$$

where $i_{1}, i_{2}, \cdots \in\{0,1, \cdots, N-1\}$.

We state the next result just for the middle-third Cantor set; but, following the discussion in $\mathbf{9}$, the reader will convince him/herself that it carries over to any fractal constructed by iteration of finite families of (contractive) affine maps in $\mathbb{R}^{d}$; so called iterated function systems (IFS). 
Proposition 4.2. Let

$$
\left\{\begin{array}{l}
m_{0}(z)=\frac{1+z^{2}}{\sqrt{2}} \\
m_{1}(z)=z \\
m_{2}(z)=\frac{1-z^{2}}{\sqrt{2}}
\end{array}\right.
$$

Then the unitarity condition (4.3) from Lemma4.1] is satisfied, and the operators

$$
\left(S_{i} f\right)(z)=m_{i}(z) f\left(z^{3}\right), f \in L^{2}(\mathbb{T}), i=0,1,2, z \in \mathbb{T},
$$

define a representation of $\mathcal{O}_{3}$ on $L^{2}(\mathbb{T})$; and the corresponding measure $\mu_{0}$ from (4.4) is the Hausdorff measure $\mu^{(s)}$ of Hausdorff dimension $s=\frac{\ln 2}{\ln 3}=\log _{3}(2)$ restricted to the middle-third Cantor set $X_{3} \subset[0,1]$.

Remark 4.3. A classical theorem of Hutchinson 11, see also 10, implies that the Cantor measure, supported on $X_{3}$, is the unique Borel probability measure $\mu$ which solves

$$
\begin{gathered}
\int f(x) d x(x)=\frac{1}{2}\left(\int f\left(\frac{x}{3}\right) d x(x)+\int f\left(\frac{x+2}{3}\right) d \mu(x)\right) \\
\text { for all continuous bounded functions } f .
\end{gathered}
$$

As a result, we need to verify that our measure $\mu_{0}$ from (4.4), and from the $\mathcal{O}_{3}$-representation, satisfies identity (4.7).

To compute the terms on the right-hand side in (4.4) is a finite matrix problem. We need only to calculate the operators $S_{i}^{*}, i=0,1,2$, on the threedimensional subspace $\mathcal{M}:=\operatorname{span}\left\{e_{0}, e_{-1}, e_{-2}\right\}$. But the argument from section 3 shows that the three restricted operators

$$
F_{i}:=\left.S_{i}^{*}\right|_{\mathcal{M}}, i=0,1,2
$$

have the following matrix representation

$$
F_{0}=\left(\begin{array}{ccc}
\frac{1}{\sqrt{2}} & 0 & 0 \\
0 & \frac{1}{\sqrt{2}} & 0 \\
0 & 0 & 0
\end{array}\right), F_{1}=\left(\begin{array}{lll}
0 & 0 & 0 \\
0 & 0 & 1 \\
0 & 0 & 0
\end{array}\right), F_{2}=\left(\begin{array}{ccc}
\frac{1}{\sqrt{2}} & 0 & 0 \\
0 & \frac{-1}{\sqrt{2}} & 0 \\
0 & 0 & 0
\end{array}\right)
$$

A substitution of (4.8)-(4.9) into (4.4) yields

$$
\begin{aligned}
& \mu_{0}\left(\left[\frac{i_{1}}{N}+\cdots+\frac{i_{k}}{N^{k}}, \frac{i_{1}}{N}+\cdots+\frac{i_{k}}{N^{k}}+\frac{1}{N^{k}}\right)\right) \\
& =\left\|F_{i_{k}} \cdots F_{i_{2}} F_{i_{1}} e_{0}\right\|^{2} \\
& =\left\{\begin{array}{l}
0 \text { if one or more of the } i_{j} \text { 's is } 1 \\
2^{-k} \text { otherwise. }
\end{array}\right.
\end{aligned}
$$

Using this, it is immediate to see that $\mu_{0}$ satisfies Hutchinson's identity (4.7), and therefore $\mu_{0}$ is the Hausdorff measure $\mu^{(s)}, s=\log _{3}(2)$, restricted to $X_{3}$. 


\section{A Technical Lemma}

In the proof of Lemma 3.6 Theorem 3.12 and Corollary 3.13 above, we relied on the following lemma regarding operators in a finite-dimensional Hilbert space. While it is analogous to the classical Perron-Frobenius theorem, our present result makes no mention of positivity. In fact, our matrix entries will typically be complex.

Notation 5.1. If $\mathcal{M}$ is a complex Hilbert space, we denote by $L(\mathcal{M})$ the algebra of all bounded linear operators on $\mathcal{M}$ If $\mathcal{M}$ is also finite-dimensional, we will pick suitable matrix representations for operators $F: \mathcal{M} \longrightarrow \mathcal{M}$ If $\mathcal{M}$ contains subspaces, $\mathcal{M}_{i}, i=1,2$ such that

$$
\mathcal{M}_{1} \perp \mathcal{M}_{2} \text { and } \mathcal{M}=\mathcal{M}_{1} \oplus \mathcal{M}_{2}
$$

then we get a block-matrix representation

$$
F=\left(\begin{array}{cc}
A & B \\
C & D
\end{array}\right)
$$

where the entries are linear operators specified as follows.

$$
A: \mathcal{M}_{1} \longrightarrow \mathcal{M}_{1}, \quad B: \mathcal{M}_{2} \longrightarrow \mathcal{M}_{1}
$$

and

$$
C: \mathcal{M}_{1} \longrightarrow \mathcal{M}_{2}, \quad D: \mathcal{M}_{2} \longrightarrow \mathcal{M}_{2}
$$

If $\operatorname{dim} \mathcal{M}_{1}=1$, and $\mathcal{M}_{1}=\mathbb{C} w$ for some $w \in \mathcal{M}$, then we will identify the operators $\mathcal{M}_{1} \longrightarrow \mathcal{M}$ with $\mathcal{M}$ via

$$
T_{\eta}: \mathbb{C} \ni z \longrightarrow z \eta
$$

where $\eta \in \mathcal{M}$. The adjoint operator is

$$
T_{\eta}^{*} x=\langle\eta \mid x\rangle w, \text { for } x \in \mathcal{M} .
$$

Lemma 5.2. Let $\mathcal{M}$ be a finite-dimensional complex Hilbert space, with $d=\operatorname{dim} \mathcal{M}$. Let $F \in L(\mathcal{M})$, and let $a \in \mathbb{C}$ satisfy the following four conditions:

(i) $a \in \operatorname{spec}(F)$;

(ii) $|a|>\max \{|\lambda| \mid \lambda \in \operatorname{spec}(F) \backslash\{a\}\}$;

(iii) the algebraic multiplicity of $a$ is one; and

(iv) there is a $w \in \mathcal{M},\|w\|=1$, such that $F^{*} w=\bar{a} w$.

Then there is a unique $\xi \in \mathcal{M}$ such that

$$
\langle w \mid \xi\rangle=1 \text { and } F \xi=a \xi .
$$

Moreover,

$$
\lim _{n \longrightarrow \infty} a^{-n} F^{n} x=\langle w \mid x\rangle \xi \text { for all } x \in \mathcal{M} .
$$

Lemma 5.3. There is a constant $C$ independent of $d=\operatorname{dim} \mathcal{M}$ and of $x$, such that

$$
\left\|a^{-n} F^{n} x-\langle w \mid x\rangle \xi\right\| \leq C n^{d-1} \max \left\{\left|\frac{s}{a}\right|^{n} \mid s \in \operatorname{spec}(F) \backslash\{a\}\right\} .
$$


Proof. [Lemma 5.2] Set

$$
\mathcal{M}^{\prime}:=\mathcal{M} \ominus \mathbb{C} w=\{x \in \mathcal{M} \mid\langle w \mid x\rangle=0\} .
$$

Then

$$
\mathcal{M}=\mathbb{C} w \oplus \mathcal{M}^{\prime},
$$

and we get the resulting block-matrix representation of $F$,

$$
F=\left(\begin{array}{cc}
a & 00 \cdots 0 \\
\eta & G
\end{array}\right)
$$

where $a$ is the number in (i), the vector $\eta \in \mathcal{M}^{\prime}$, and operator $G \in L\left(\mathcal{M}^{\prime}\right)$, are uniquely determined. As a result, we get the factorization

$$
\operatorname{det}(\lambda-F)=(\lambda-a) \operatorname{det}(\lambda-G)
$$

for the characteristic polynomial. Assumptions (ii) and (iii) imply

$$
\operatorname{spec}(F) \backslash\{a\}=\operatorname{spec}(G) ;
$$

and in particular, we note that $a$ is not in the spectrum of $G$. Hence the inverse $(a-G)^{-1}$ is well defined, and $(a-G)^{-1} \in L\left(\mathcal{M}^{\prime}\right)$. We claim that the vector

$$
\xi=w+(a-G)^{-1} \eta
$$

satisfies the conditions in (5.2). First note that $(a-G)^{-1} \eta \in \mathcal{M}^{\prime}$, so $\langle w \mid \xi\rangle=$ $\langle w \mid w\rangle=\|w\|^{2}=1$. Moreover,

$$
F \xi=a w+\eta+G(a-G)^{-1} \eta=a w+a(a-G)^{-1} \eta=a \xi,
$$

which proves the second condition in (5.2). Uniqueness of the vector $\xi$ in (5.2) follows from (5.8). Using the matrix representation (5.6), we get

$$
F^{2}=\left(\begin{array}{cc}
a^{2} & 00 \cdots 0 \\
a \eta+G \eta & G^{2}
\end{array}\right)
$$

and by induction,

$$
\begin{aligned}
F^{n} & =\left(\begin{array}{cc}
a^{n} & 00 \cdots 0 \\
a^{n-1} \eta+a^{n-2} G \eta+\cdots+G^{n-1} \eta & G^{n}
\end{array}\right) \\
& =\left(\begin{array}{cc}
a^{n} & 00 \cdots 0 \\
\left(a^{n}-G^{n}\right)(a-G)^{-1} \eta & G^{n}
\end{array}\right) .
\end{aligned}
$$

Hence, if we show that

$$
\lim _{n \longrightarrow \infty} a^{-n} G^{n}=0,
$$

then the desired conclusion (5.3) will follow. Using the matrix form (5.10), the conclusion (5.3) reads

$$
\lim _{n \longrightarrow \infty} a^{-n} F^{n}=\left(\begin{array}{cc}
1 & 00 \cdots 0 \\
(a-G)^{-1} \eta & 0
\end{array}\right) .
$$

In proving (5.11), we will make use of the Jordan-form representation for $G$. Jordan's theorem applied to $G$ yields three operators $D, V, N \in L\left(\mathcal{M}^{\prime}\right)$ with the following properties: 
(1) $D$ is a diagonal matrix with the numbers $\operatorname{spec}(F) \backslash\{a\}$ down the diagonal;

(2) $V$ is invertible;

(3) $N$ is nilpotent: If $d-1=\operatorname{dim}\left(\mathcal{M}^{\prime}\right)$ then $N^{d-1}=0$;

(4) $[N, D]=N D-D N=0$;

(5) $G=V(D+N) V^{-1}$

Let $x \in \mathcal{M}^{\prime}$, and let $n \geq d$. Using (2)-(5), we get

$$
\begin{aligned}
a^{-n} G^{n} x & =V a^{-n}(D+N)^{n} V^{-1} x \\
& =\sum_{i=0}^{d-2}\left(\begin{array}{c}
n \\
i
\end{array}\right) V a^{-n} D^{n-i} N^{i} V^{-1} x .
\end{aligned}
$$

But the matrix $a^{-n} D^{n-i}$ is diagonal with entries

$$
\left\{a^{-n} s^{n-i} \mid s \in \operatorname{spec}(F) \backslash\{a\}\right\}, 0 \leq i \leq d-1 .
$$

Using finally assumption (ii), we conclude that

$$
\lim _{n \longrightarrow \infty}\left(\begin{array}{c}
n \\
i
\end{array}\right) a^{-n} s^{n-i}=0,
$$

and the proof of (5.11) is completed.

Proof. [Lemma 5.3] Let the conditions be as stated in the Remark. From the arguments in the proof of Lemma 5.2 we see that the two vectors on the left-hand side in (5.4) may be decomposed as follows:

$$
a^{-n} F^{n} x=\langle w \mid x\rangle w+\left(1-a^{-n} G^{n}\right)(a-G)^{-1} \eta+a^{-n} G^{n} P_{\mathcal{M}^{\prime}} x ;
$$

and

$$
\langle w \mid x\rangle \xi=\langle w \mid x\rangle w+(a-G)^{-1} \eta .
$$

Hence, the difference is in $\mathcal{M}^{\prime}$, and

$$
\begin{aligned}
& \left\|a^{-n} F^{n} x-\langle w \mid x\rangle \xi\right\| \\
& =\left\|a^{-n} G^{n}\left(P_{\mathcal{M}^{\prime}} x-(a-G)^{-1} \eta\right)\right\| \\
& \leq C n^{d-1} \max \left\{\left|\frac{s}{a}\right|^{n} \mid s \in \operatorname{spec}(F) \backslash\{a\}\right\}
\end{aligned}
$$

which is the desired conclusion (5.4).

\section{References}

[1] L. W. Baggett, P. E. T. Jorgensen, K. D. Merrill, J. A. Packer, An Analogue of BratteliJorgensen Loop Group Actions for GMRA's, Vol. 345 of Wavelets, frames and operator theory, Contemp. Math., , American Mathematical Society, Providence, RI, 2004, pp. $11-25$.

[2] O. Bratteli, D. E. Evans, P. E. T. Jorgensen, Compactly Supported Wavelets and Representations of the Cuntz Relations, Appl. Comp. Harmon. Anal., 8 (2000) 166-196.

[3] O. Bratteli, P. E. T. Jorgensen, Wavelets through a Looking Glass: The World of the Spectrum, Applied and Numerical Harmonic Analysis, Birkhäuser, Boston, 2002.

[4] O. Bratteli, P. E. T. Jorgensen, Iterated function systems and permutation representations of the Cuntz algebra, Mem. Amer. Math. Soc. 139 (1999) no. 663. 
[5] R. Coifman, Y. Meyer, V. Wickerhauser, Size properties of wavelet-packets, in: M. Ruskai, G. Beylkin, R. Coifman, I. Daubechies, S. Mallat, Y. Meyer, L. Raphael (Eds.), Wavelets and Their Applications, Jones and Bartlett, Boston, 1992, pp. 453-470.

[6] J. Cuntz, Simple $C^{*}$-algebras generated by isometries, Comm. Math. Phys. 57 (1977) $173-185$.

[7] I. Daubechies, Ten lectures on wavelets, CBMS-NSF Regional Conference Series in Applied Mathematics, Philadelphia: Society for Industrial and Applied Mathematics,Vol. 61, 1992.

[8] D. E. Dutkay; P. E. T. Jorgensen, Wavelet constructions in non-linear dynamics. Electron. Res. Announc. Amer. Math. Soc. 11. (2005), 21-33 (electronic).

[9] D. Dutkay, P. Jorgensen, Wavelets on Fractals, preprint, University of Iowa, 2004, arXiv:math.CA/0305443 accepted: Rev. Mat. Iberoamericana.

[10] K. J. Falconer, The Geometry of Fractal Sets, Cambridge, Tracts on Mathematics, 85.

[11] J. E. Hutchinson, Fractals and self-similarity, Indiana Univ. Math. J. 30 (1981) 713-747.

[12] P. E. T. Jorgensen; D. W. Kribs, Wavelet representations and Fock space on positive matrices. J. Funct. Anal. 197 (2003), no. 2, 526-559.

[13] P.E.T. Jorgensen, Minimality of the Data in Wavelet Filters, Advances in Mathematics 159, 2001, pp. 143-228.

[14] P.E.T. Jorgensen, Measures in wavelet decompositions, Adv. Appl. Math., Vol. 23 (2005), 561-590.

[15] M. V. Wickerhauser, Best-adapted wavelet packet bases, in: I. Daubechies (Ed.), Different Perspectives on Wavelets (San Antonio, TX, 1993), Vol. 47 of Proc. Sympos. Appl. Math., American Mathematical Society, Providence, 1993, pp. 155-171.

[16] M. V. Wickerhauser, Adapted Wavelet Analysis from Theory to Software, IEEE Press, New York, A.K. Peters, Wellesley, MA, 1994.

Department of Mathematics, The University of Iowa, 14 Maclean Hall, Iowa City, IA, 52242-1419, U.S.A.

E-mail address: http://www.math.uiowa.edu/ jorgen 

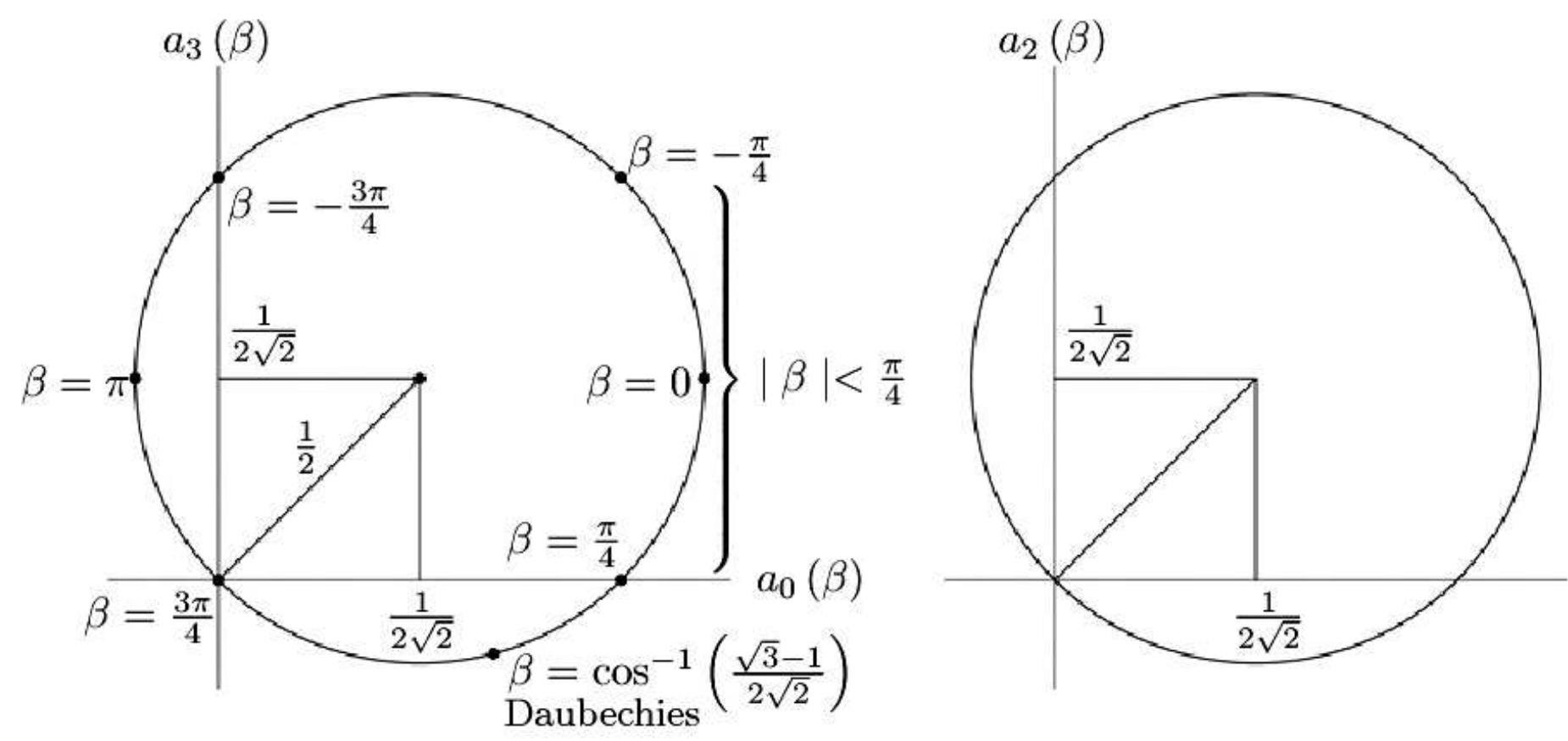

$a_{1}(\beta)$ 


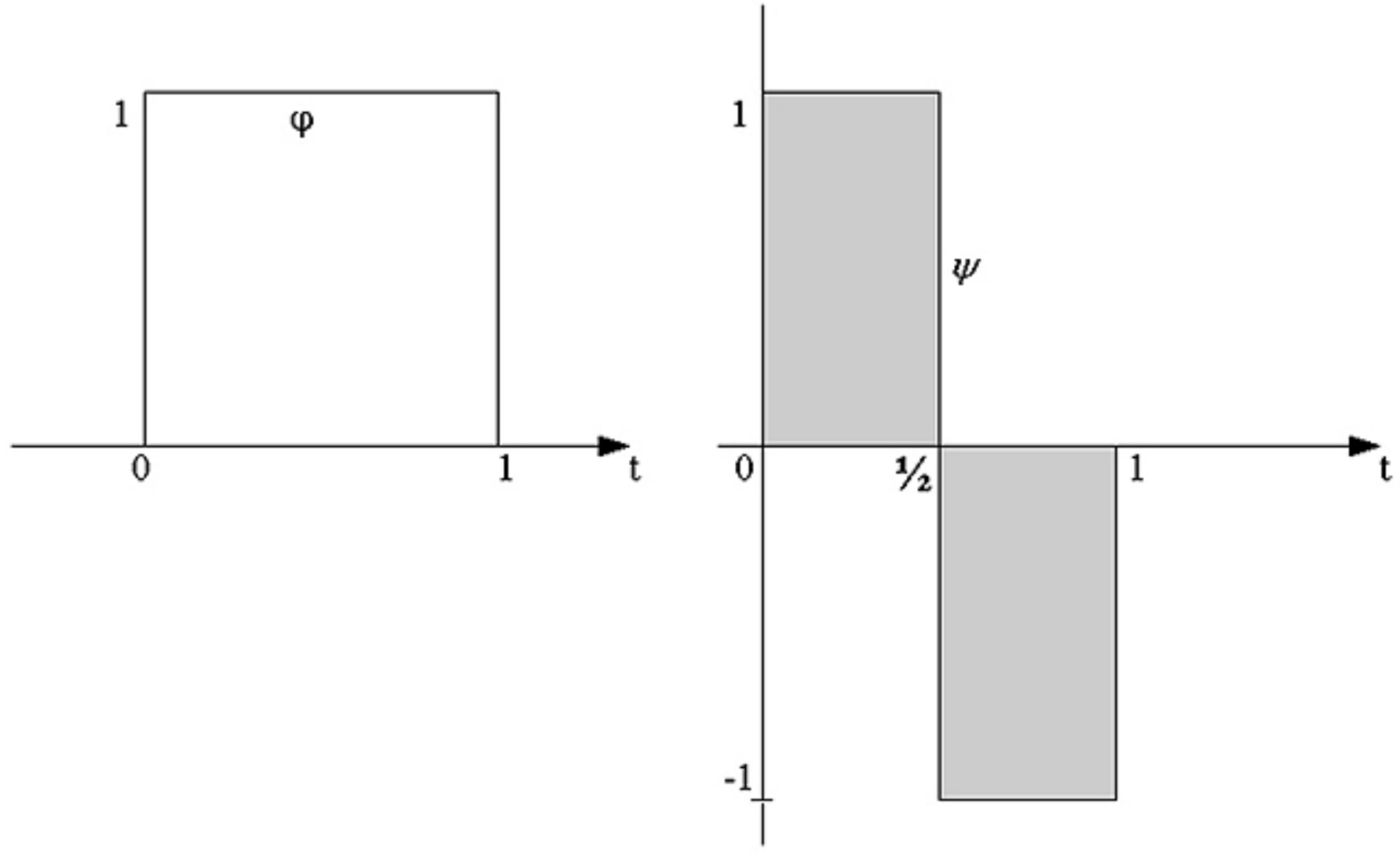

